\section{Pupillary responses to single and sinusoidal light stimuli in diabetic patients}

\author{
Wolfgang $\mathrm{H}$. Zangemeister, Thilo \\ Gronow, Ulrich Grzyska' \\ Departments of Neurology and \\ 'Neuroradiology, University of Hamburg, \\ Germany
}

\section{Abstract}

We examined effects of diabetes mellitus (DM) on the pupillary light reflex (PLR). Phasic pupillary response to a single light stimulus (200 ms) (pPLR) and to continuous sinusoidal stimuli with four different frequencies $(0.1,0.3,0.7,1.3 \mathrm{~Hz})$ (cPLR) were examined in $52 \mathrm{DM}$ patients and 21 control subjects. We asked: does recording and frequency analysis of cPLR together with short time fourier [STFT] analysis of pPLR differentiate better between DM patients and normal subjects than pPLR only?

Initial pupil diameter was significantly decreased in the DM group. For pPLR. maximal contraction velocity (Vmax), Vmax of redilation 1, reflex-amplitude and pPLR latency were significantly reduced in those patients who also showed signs of diabetic autonomic neuropathy (DNP). Tests of dynamic pupillary light reflex (cPLR) revealed that all DM patients had a significantly reduced gain at lower frequencies. Pupil phase lag was greater at 0.1 and $0.3 \mathrm{~Hz}$ and smaller at 0.7 and $1.3 \mathrm{~Hz}$ in the DNP group $(p<0.001)$. Comparison of single pPLR recordings of 5 DNP patients with 5 subjects using short time fast fourier (STFT) analysis revealed a characteristic change from low frequency content in healthy subjects to high frequency content in DNP patients.

Significant changes in the PLR in DM can be found only when symptoms of autonomic neuropathy have been shown. Both sympathetic and the parasympathetic nervous systems are affected by diabetic autonomic neuropathy. Only recording of cPLR, together with STFT of pPLR can identify significant pathological deficits of pupillary control in single cases.

\section{Introduction}

Studies of the autonomic nervous system have demonstrated the effect of diabetic neuropathy on heart frequency and regulation of blood pressure to orthostatic changes. As the size and movement of the pupil is relatively easy to record, many studies have tried to eval- uate different pupil parameters and link these results with the clinical course of the autonomic neuropathy.

Also, pupillary reactions to light stimulation that were modulated in time were recorded by many previous authors. ${ }^{1-10}$ They found that in patients with diabetes mellitus the critical flicker fusion frequency was decreased when pupillary reactions to light stimulation are recorded, i.e. the rate of presentation of intermittent, alternate, or discontinuous photic stimuli that just gives rise to a fully uniform and continuous sensation obliterating flicker that permits phase and gain of the actual pupil to be judged.

The aim of our study was to differentiate pupillary response abnormalities in diabetics that are linked to the autonomic nervous system disease from those which are the consequence of the initial condition of the stimulated pupil.

\section{Materials and Methods}

\section{Patients}

We examined 52 patients with diabetes mellitus (21 females, 31 males, median age 46.7 years, range 19-74 years). Twenty-seven patients had a type I diabetes and 24 a type II diabetes. The average duration of the disease was 9.2 years (range 1-44 years). All patients were regularly examined in the diabetes unit of the Medical University Hospital, Hamburg. None of these patients were taking drugs during the period of the study which were known to influence the pupil or the autonomic nervous system. The control group consisted of 21 healthy subjects ( 10 females, 11 males, median age 46.2 years, range $17-73$ years). The distribution of age and sex were almost identical. Ophthalmological check-up excluded a retinopathy and rubeosis iridis. All participants were informed about the purpose and goal of the study before it started and gave their informed consent.

\section{Technical methods}

For pupil recordings we used a compact integrated pupillograph (AMTech Company, Weinheim, Germany). The instrument was focused on the iris with a distance of approximately $5 \mathrm{~cm}$ between front lens and iris, where light was excluded by a rubber tube. The direct light reflex of this eye was recorded. The fellow eye fixated a distant target at infinity. The pupillary recording device was positioned directly in front of the eye that was recorded linked through a dark rubber tube. The recording was made in reduced room light (near mesopic conditions). While the case history was recorded and a neurological check-up was performed, the subject's eyes could adapt to the reduced room light. The position and focus
Correspondence: Wolfgang H. Zangemeister, Neurological University Clinic, Martinistrasse 52-010, D-20251 Hamburg, Germany.

E-mail:whiz@uke.uni-hamburg.de.

Acknowledgements: for critical discussions and improvements of this paper in an earlier stage we are indebted to the late Prof. Lawrence Stark from UC Berkeley.

Key words: pupil, infrared reflex pupillography, autonomic neuropathy, diabetes mellitus, neuroophthalmology, sinusoidal light stimuli to the pupil, short time FFT analysis.

Received for publication: 12 July 2009.

Revision received: 4 September 2009.

Accepted for publication: 28 September 2009.

This work is licensed under a Creative Commons Attribution 3.0 License (by-nc 3.0).

(C) Copyright W.H. Zangemeister et al., 2009 Licensee PAGEPress, Italy

Neurology International 2009; 1:e19

doi:10.4081/ni.2009.e19

was controlled by an electronic viewfinder on the back of the camera (2.5 inch LCD screen).

The phasic light stimulus was generated by a light emitting diode with an intensity of $10,000 \mathrm{~cd} / \mathrm{m}^{2}$, a duration of $200 \mathrm{msec}$, and a wave length of $585 \mathrm{~nm}$. The recording of the phasic pupillary light reflex (pPLR) started with the stimulus and was stopped after 2 seconds. The response including calculated dynamics was stored on a PC (Figure 1).

The software calculated values for velocities of contraction, of maximal dilation 1 and 2, the initial pupillary diameter, the latency time (between stimulus onset and start of contraction, determined by linear curve fits), and the time to peak meiosis given by the point of maximal contraction. The velocity of dilation 1 showed the maximum steepness of the response curve within the interval of the lower $20 \%$ and the upper $80 \%$ of the final diameter, and the velocity of dilation 2 between the lower $35 \%$ and the upper $95 \%$ of the final pupil diameter. For each eye, we evaluated at least 3 artifact free measurements. Velocity of dilation 1 reflects the general difference between parasympathetic and sympathetic influences on the pupil, whereas velocity of dilation 2 is believed to reflect solely the sympathetic influence on pupil dilation,

Besides the pupillary recordings, other typical autonomic variables were checked: heart rate during in- and expiration, the changes in blood pressure and heart frequency when standing up after a lying position. Vibration, touch and pain were tested in the distal extremities for subjective decreases in sensation, suggestive of neuropathy.

After a short break, the dynamic pupillary 
reflex (cPLR) was recorded for the frequencies $0.1,0.3,0.7$ and $1.3 \mathrm{~Hz}$. As lid movements were often interfering, especially with the lowest frequency, we recorded at least 3 samples of each frequency. The highest intensity of these sinusoidal stimuli was again $10,000 \mathrm{~cd} / \mathrm{m}^{2}$, the lowest $1,000 \mathrm{~cd} / \mathrm{m}^{2}$.

To improve the differentiation between patients with two signs of diabetic neuropathy $(n=23)$, (i.e. two out of three parameters: heart rate, decrease of distal sensation, change in blood pressure) and other patients with no such signs $(n=29)$ we compared this subgroup with the normal group which showed a lower age (median age of the PNP group $59.7 \pm 10.1$ vs. $46.2 \pm 14.5$ control). The fraction of meiosis with the initial pupil diameter that was due to the age of the patient would be accounted for by the addition of $0.04 \mathrm{~mm}$ per year to the recorded initial pupil diameter. This reflects the average decrease of pupil diameter per year of life (2.5); so we used an age corrected initial pupil diameter (ACPD).

Since there were no reliable data for the other parameters of the pupillary light reflex, we could not use the same procedure for these dynamical values which would have been more appropriate with respect to the final evaluation of our findings. The dynamic pupil data were first analyzed using a fast fourier analysis (FFT; Autosignal 1.52) and than statistically evaluated. Data analysis was performed with the "Statistika" program; we used Student's ttest for group comparisons, or in case there was no normal distribution, we used the Mann-Whitney rank sum test. For the calculation of correlations we used the Pearson product moment value.

Additionally, we analyzed the pPLR of 5 DM patients with signs of autonomic neuropathy applying short time fourier transformation analysis (STFT) (program auto-signal.1.52) and compared these with the pPLR of 5 normal subjects using the Mann-Whitney rank sum test.

\section{Results}

The initial pupil diameter was the only parameter significantly decreased in the diabetic patients: $4.85 \pm 0.97 \mathrm{~mm}$ versus $5.38 \pm 0.87$ $\mathrm{mm}(p<0.005)$ (Table 1 and Figure 2). It is important to note that because of the age factor leading to smaller pupils for over 60 years of age the discrimination between diabetics and normal subjects by pupil size becomes less evident, but is still significant with $p<0.02$.

All other parameters showed no statistical difference to the control group. The subgroup of patients with diabetes mellitus who showed at least two other signs of diabetic neuropathy showed an even more significant difference

\section{Pupil Light Reflex (PLR) 18.09.2007 11:27}

E. M. * 08.01.1949

18.09.2007. 11:27. RE

Latency

Duration of response

Amplitude

Initial diameter

Relative amplitude

Velocity of constriction

$2 / 3$ of constriction duration

$2 / 3$ of constriction time point

$1 / 3$ of redilation duration

$1 / 3$ of redilation time point

Velocity of dilatation fast

Velocity of dilatation slow

Steepness of plateau

Begin of plateau

End of plateau

Begin velocity of constriction

End velocity of constriction

Begin V-Dil fast

End V-Dil fast

Begin V-Dil slow

End V-Dil slow

Time of minimum

Quality rating

Stimulus-brightness

Stimulus-begin

Stimulus-end

Date

Time

$0.299 \mathrm{sec}$

$0.369 \mathrm{sec}$

$0.740 \mathrm{~mm}$

$4.040 \mathrm{~mm}$

$18.310 \%$

$2.930 \mathrm{~mm} / \mathrm{sec}$

$0.168 \mathrm{sec}$

$0.468 \mathrm{sec}$

$0.308 \mathrm{sec}$

$0.976 \mathrm{sec}$

$1.300 \mathrm{~mm} / \mathrm{sec}$

$0.390 \mathrm{~mm} / \mathrm{sec}$

$0.010 \mathrm{~mm} / \mathrm{sec}$

$0.004 \mathrm{sec}$

$0.284 \mathrm{sec}$

$0.332 \mathrm{sec}$

$0.444 \mathrm{sec}$

$0.896 \mathrm{sec}$

$0.992 \mathrm{sec}$

$1.392 \mathrm{sec}$

$1.696 \mathrm{sec}$

$0.668 \mathrm{sec}$

$\mathrm{OK}$

high 200

$0.000 \mathrm{sec}$

$0.300 \mathrm{sec}$

18.09 .01

11:28:32

Measuring mode

CIP Version

PLR

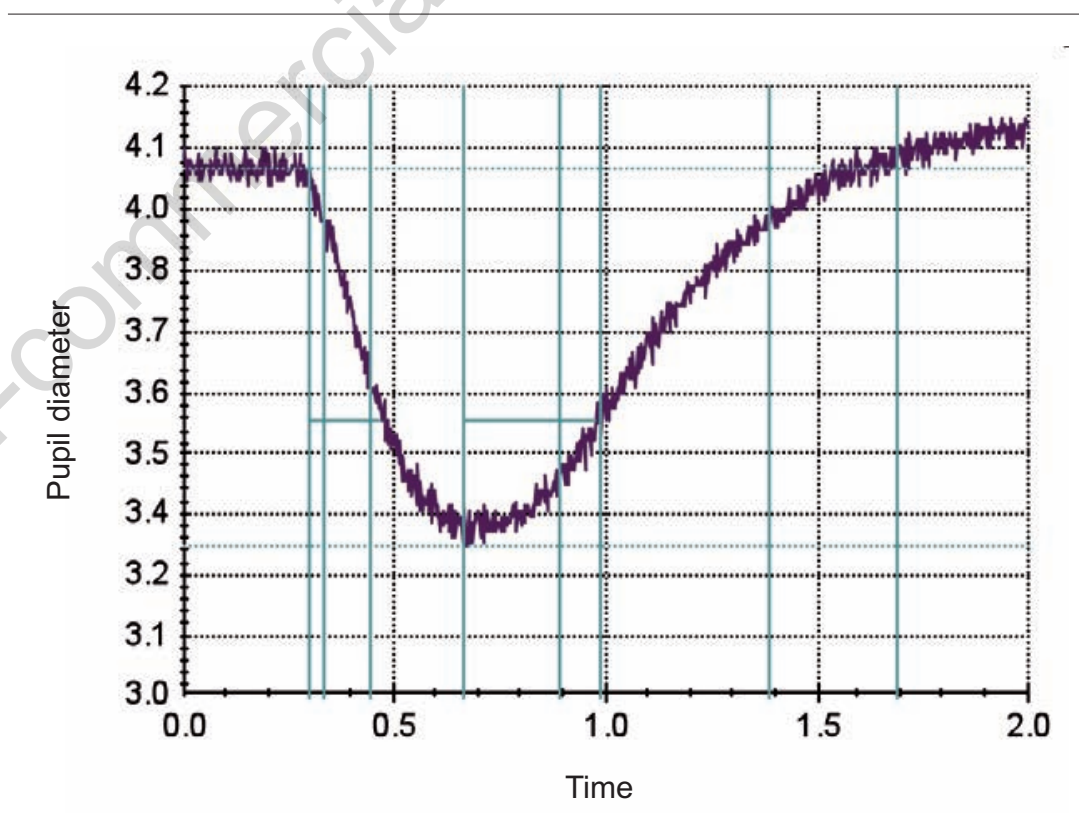

Figure 1. Example printout of pPLR time and dynamic variables and pPLR original.

with respect to the pupil diameter: $4.63 \pm 0.69$ $\mathrm{mm}(p<0.001)$ (Table 1).

Also, in this group the phasic PLR dynamics were reduced: the maximum contraction velocity was $3.44 \pm 1.28 \mathrm{~mm} / \mathrm{sec}$ versus $4.42 \pm 0.93$ $\mathrm{mm} / \mathrm{sec}(p<0.01)$, the first velocity of dilation was also decreased with $0.99 \pm 0.26 \mathrm{~mm} / \mathrm{sec}$ versus $1.18 \pm 0.28 \mathrm{~mm} / \mathrm{sec}(p<0.01)$, the second velocity of dilation $0.42 \pm 0.21 \mathrm{~mm} / \mathrm{sec}$ versus $0.52 \pm 0.19 \mathrm{~mm} / \mathrm{sec}(p<0.05)$, the amplitude of contraction was $1.26 \pm 0.45 \mathrm{~mm}$ versus $1.53 \pm 0.34 \mathrm{~mm}(p<0.01)$. In the patient subgroup with additional signs of autonomic neuropathy, the latency was increased: $0.27 \pm 0.04$ 
sec versus $0.25 \pm 0.03 \mathrm{sec}(p<0.05)$. The overall group of patients with diabetes mellitus demonstrated similar values as the subgroup, but the differences to the normal group values did not reach significance.

When normalized to the decreased initial pupil diameter as a sign of the different initial conditions of the pupil compared to the normal pupil, the relative reflex amplitude as the percentual amplitude of the initial pupil diameter did not show significant differences in any group: $31.2 \pm 8.59 \%$ (all patients) versus $30.6 \pm 9.09 \%$ (patients with DNP) versus $29.1 \pm 7.64 \%$ (control group). From this fact it is obvious that in patients with DM any pupil of a given diameter shows in many respects similar dynamics to a normal pupil. For the complete DM group, this is reflected in the values of the relative dynamics: contraction velocity (\%): $83.6 \pm 27.8$ in the diabetes group versus $84.2 \pm 21.4$ in the control group; velocity of dilation 1 (\%): $24.2 \pm 6.33$ in the patient group versus $24.3 \pm 5.13$ in the control group; velocity of dilation 2 (\%): $9.8 \pm 4.25$ in the patient group versus $9.91 \pm 3.71$ in the control group. For the DNP group, however, the relative contraction velocity was significantly decreased: $76.5 \%$ versus $85.4 \%$ as well as the relative dilation velocity 1 . We found a highly significant correlation between the amplitude of the pPLR and the initial pupil diameter $(\mathrm{r}=0.50, p<0.001)$. Also, we found significantly positive correlations between the single velocity parameters checked and also between the velocity parameters and the initial pupil diameter $(\mathrm{r}=0.5$, $p<0.01$ ). These correlations were found within all groups. The latency did not show a very significant correlation with the initial pupil diameter or the velocities $(p<0.04)$, neither in the diabetes group nor in the control group. The initial pupil diameter showed an inverse correlation with age $(p<0.001)$.

After a short break, the dynamic pupillary reflex was recorded for the frequencies 0.1 , 0.3, 0.7 and 1.3 Hz. (Tables 2 and 3).

In the DNP patients' group, who showed at least two clinical signs of neuropathy, the pupil gain was highly significantly diminished, especially for lower frequencies, whereas in the overall group only for lower frequencies were some differences found.

The pupil phase of the patients varied with respect to the control group. For the lower frequencies of 0.1 and $0.3 \mathrm{~Hz}$ in the DNP patients we found a highly significant phase lead. This showed up only as a tendency within the group of all patients. For higher frequencies of 0.7 and $1.3 \mathrm{~Hz}$ we found a significant phase lag, especially in the DNP patients. Our comparison of the frequency distribution over time using short time fourier transformation analysis (STFT) showed the abnormal decrease in low frequency variability in DM patients' phasic pupillary reflex as seen in Figure 3 and the increase in higher frequency content, probably due to greater stiffness of the iris muscle for both biomechanical and neural control reasons.

The time-frequency spectrum could be plotted in a variety of formats. The most useful options for these spectra were the $\mathrm{dB}$ formats for visualization. We have used contour plots of normalized decibels: Re is the real component of the continuous wave transformation at a given time and frequency, Im is the imaginary component, $n$ is the data set size: $d B$. decibels. $10.0 * \log 10\left(\operatorname{Re}^{*} \operatorname{Re}+\operatorname{Im} * \operatorname{Im}\right)$, normalized to 0 for time-frequency node with maxi-

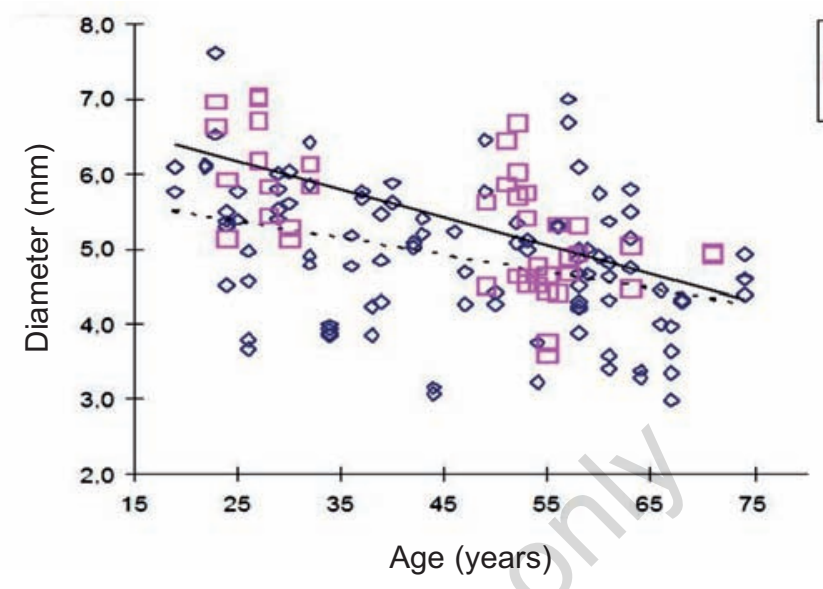

$\diamond$ Diabetics

$\square$ Control

Group

Figure 2. Pupil size as function of age in normal subjects and DM patients. Note the smaller size in DM patients in spite of large variance. Continuous line: linear fit to normal results; dashed line: linear fit to data of DM patients. The fraction of meiosis with the initial pupil diameter that was due to the age of the patient would be accounted for by the addition of $0.04 \mathrm{~mm}$ per year to the recorded initial pupil diameter. This reflects the average decrease of pupil diameter per year of life; so we used an age corrected initial pupil diameter (ACPD). It is important to note that because of the age factor leading to smaller pupils over 60 years of age the discrimination between diabetics and normal subjects by pupil size becomes less evident, but is still significant with $p<0.02$.

Table 1. Comparison of all relevant phasic pupil dynamic parameters of the 3 groups.

\begin{tabular}{lccc} 
Parameter & $\begin{array}{c}\text { All Patients } \\
(\mathrm{n}=52)\end{array}$ & $\begin{array}{c}\text { Control Group } \\
(\mathrm{n}=21)\end{array}$ & $\begin{array}{c}\text { Patients with DNP } \\
(\mathrm{n}=23)\end{array}$ \\
PD initial (mm) A* & $4.85 \pm 0.96^{* *}$ & $5.38 \pm 0.87$ & $4.63 \pm 0.69^{* * *}$ \\
Amplitude contr. (mm) & $1.49 \pm 0.41$ & $1.53 \pm 0.34$ & $1.26 \pm 0.45^{*}$ \\
\hline Rel. Amplitude con.(\%) & $31.0 \pm 8.59$ & $29.1 \pm 7.64$ & $30.6 \pm 9.02$ \\
Vmax contr. (mm/s) & $4.16 \pm 1.22$ & $4.42 \pm 0.93$ & $3.44 \pm 1.28^{* * *}$ \\
\hline Rel. Vmax contr. (in \%) & $85.4 \pm 21.9$ & $84.2 \pm 24.4$ & $76.5 \pm 21.2^{* *}$ \\
Vmax dil 1 (mm/s) & $1.12 \pm 0.26$ & $1.18 \pm 0.28$ & $0.99 \pm 0.26^{*}$ \\
\hline Rel. Vmax dil 1 (in \%) & $23.3 \pm 5.52$ & $22.3 \pm 5.13$ & $21.2 \pm 6.33^{*}$ \\
Vmax dil 2 (mm/sec) & $0.55 \pm 0.49$ & $0.52 \pm 0.19$ & $0.42 \pm 0.21^{*}$ \\
\hline Rel. Vmax dil 2 (in \%) & $9.98 \pm 4.38$ & $9.91 \pm 3.71$ & $9.80 \pm 4.25$ \\
t min (sec) & $0.83 \pm 0.09$ & $0.81 \pm 0.07$ & $0.84 \pm 0.09$ \\
\hline Latency (sec) & $0.27 \pm 0.09$ & $0.25 \pm 0.03$ & $0.28 \pm 0.04^{*}$ \\
Reaction Time (sec) & $0.57 \pm 0.11$ & $0.58 \pm 0.23$ & $0.57 \pm 0.11$ \\
\hline
\end{tabular}

${ }^{*} p<0.01 ;{ }^{* *} p<0.001 ;{ }^{* * *} p<0.0001$ significant difference to control group. \pm standard deviation. $\mathrm{A}^{*}$ :age dependent normal pupil diameter; relrelative.

Table 2. Dynamic pupil gain.

\begin{tabular}{lccc} 
Group & Control Group & Patients with DNP & All Patients \\
Frequency Hz & $\mathbf{N}=21$ & $\mathbf{N}=23$ & $\mathbf{N}=52$ \\
0.1 & $0.186[0.07]$ & $0.158[0.06]^{* * *}$ & $0.156[0.08]^{* *}$ \\
0.3 & $0.156[0.04]$ & $0.134[0.05]^{* * *}$ & $0.145[0.06]^{*}$ \\
\hline 0.7 & $0.121[0.04]$ & $0.109[0.05]^{* *}$ & $0.118[0.06]$ n.s. \\
1.3 & $0.068[0.02]$ & $0.051[0.03]^{* *}$ & $0.065[0.07]$ n.s. \\
\hline
\end{tabular}

${ }^{*} p<0.01 ; * *{ }^{*} p<0.001 ; * * *{ }^{*} p<0.0001$ significant difference to control group.. standard deviation $=[$ ]; n.s. no significant difference to control group. 
mum power. In conjunction with the Spectrum there are 24 contour types; this means that a different color will be used for each $1 \mathrm{~dB}$ delta in the spectrum, with light cyan at the low end and dark red at the high end.

\section{Summary of results}

All DM patients showed the following group differences compared to healthy subjects:

- pupil diameter highly significantly smaller in DM patients

- cPLR gain for low frequencies lower only in DNP patients, but not so in all DM patients

- cPLR phase lag, for low phase lead for high frequency in all DM patients

DM patients with neuropathy [DNP] showed additional differences:

- pPLR Vmax contraction significantly smaller in DNP

- pPLR contraction amplitude smaller in DNP

- pPLR Vmax dilation 1 smaller in DNP

- pPLR larger amount of high frequency content during contraction (short time FFT) in DNP

- cPLR for low frequencies phase lag, for high frequencies phase lead in DNP

- cPLR gain for low frequencies phase lag, for high frequencies phase lead in DNP

Table 4 summarizes the significancy of all recorded variables that discriminate $\mathrm{DM}$ patients from the normal group; three out of seven parameters (pupil diameter, gain and phase) distinguish all DM patients from healthy subjects. Therefore, the cPLR appears to be very important for this differentiation.

DNP patients with an additional two signs of neuropathy are discriminated from the rest with very high significancy through all seven parameters. This means that the application of both the cPLR and the pPLR is the favorable combined measurement to distinguish especially the DM patients with vegetative neuropathy.

The following case exemplifies our main findings (Figure 4B) and demonstrates a typical location of mesoencephalic and pontine lacunes (Figure 4A) due to a diabetic microangiopathy. This is not very frequently found as Keane and others have stated before; ${ }^{11}$ however, if present, ${ }^{10,12}$ it is a sign of a high level pupillary control deficit as opposed to the more peripheral factors (see Discussion).

\section{Discussion}

One expected result of our study was the decreased initial pupil size in the diabetes group, in particular in those patients with additional signs of autonomic neuropathy.

Table 3. Dynamic pupil phase $\left(^{\circ}\right)$.

\begin{tabular}{lccc} 
Group & Control Group & Patients with DNP & All Patients \\
Frequency Hz & $\mathrm{N}=21$ & $\mathrm{~N}=23$ & $\mathrm{~N}=52$ \\
0.1 & $-3.10[13.75]$ & $54.1[14.2]^{* * *}$ & $7.6[17.19]^{*}$ \\
0.3 & $30.4[14.38]$ & $71.3[13.6]^{* * *}$ & $35.8[13.3]$ n.s. \\
\hline 0.7 & $108.3[15.34]$ & $98.3[14.6]^{* *}$ & $104.7[17.61]$ n.s. \\
1.3 & $208.2[17.14]$ & $141.4[18.6]^{* * *}$ & $178.7[19.02]^{* *}$
\end{tabular}

${ }^{*} p<0.01 ;{ }^{* *} p<0.001 ;{ }^{* * *} p<0.0001$ significant difference to control group. \pm standard deviation= [ ]; n.s. no significant difference to control group.

Table 4. Summary of the significancy of all recorded variables that discriminate DM patients from the normal group; out of seven parameters three, pupil diameter, gain and phase, distinguish all DM patients from healthy subjects. Therefore, the cPLR appears to be very important for this differentiation. Npst.: in column "frequency content", we analyzed the total frequency content of three pPLR each for the same time span of $\mathbf{4 0 0}$ msec. Then we had to use a non-parametric statistical test (the Mann-Whitney rank sum test) for comparison of 5 normal with 5 DNP patients' results.

\begin{tabular}{llllllll} 
& $\begin{array}{l}\text { Pupil } \\
\text { Diameter }\end{array}$ & $\begin{array}{l}\text { Vmax } \\
\text { Contract }\end{array}$ & $\begin{array}{l}\text { Vmax } \\
\text { Dilation1 }\end{array}$ & $\begin{array}{l}\text { Amplitude } \\
\text { Contract }\end{array}$ & $\begin{array}{l}\text { Frequency } \\
\text { Content }\end{array}$ & Gain & Phase \\
DNP & 0.0001 & 0.0001 & 0.01 & 0.01 & 0.01 & 0.0001 low F & 0.0001 \\
N=21 & & & & & npst. & 0.01 high F & \\
All DM & 0.001 & n.s. & n.s. & n.s. & n.s. & 0.01 low F & 0.01 \\
N=52 & & & & & & n.s. high F & 0.001 \\
\hline
\end{tabular}

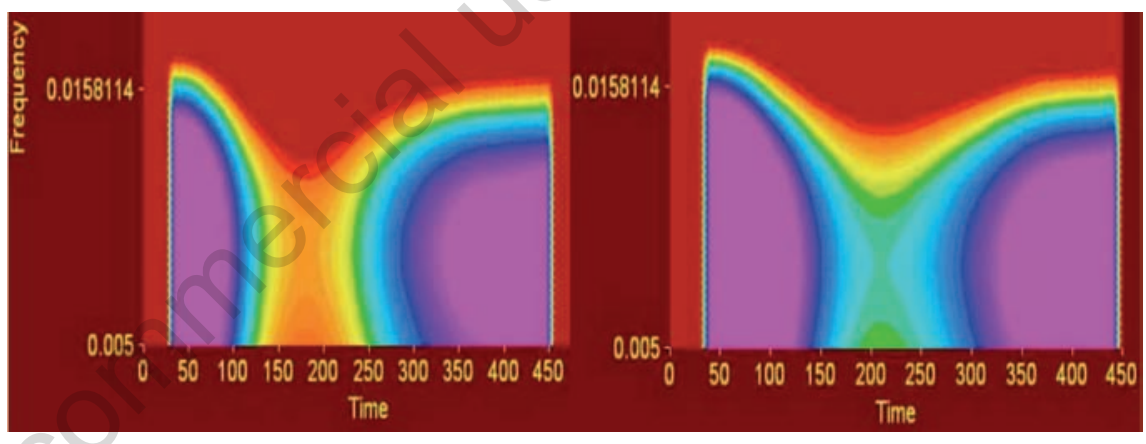

Figure 3. pPLR single response from the left eye of a diabetic patient with signs of DNP (left) compared to a normal subject (right), using short time fourier transformation (STFT) as function of time plot. A significantly larger amount of high frequency content during both pPLR phases, contraction and dilation, is shown.

This is in agreement with other studies which have analyzed the phasic pupillary light reflex in diabetic patients. ${ }^{13-15}$ To what extent did dynamic parameters of the pPLR depend on this decreased initial pupil size?

The pPLR amplitude, as well as the velocities of contraction and dilation 1 and 2, were decreased in our study in the overall group of patients, and in particular in the subgroup which included at least two signs of autonomic neuropathy. However, this difference could not be found in patients with no signs of autonomic neuropathy after normalizing these values with respect to the initial relative pupil size. We conclude that only high resolution short time FFT may permit a better insight into the changes in individual pupil dynamics due to DM (Figure 3), whereas the time functions permit this only in DM patients with neuropathy. In this respect, we were not able to confirm exactly the values of contraction and velocities that have been reported by Straub et al. ${ }^{16}$ This was probably due to the fact that Straub et al. measured their contraction velocity during one second and not at the peak of the contraction velocity.

\section{Latencies, gain and phase}

Niakan et al. ${ }^{17}$ Pfeiffer et al. ${ }^{11}$ and Lanting et $a l .{ }^{18,19}$ reported that the latencies of the pPLR in patients with diabetes mellitus were significantly increased; they concluded that the increased latencies were an early sign of neuropathy. In our study, similar to the studies by Hreidarrson et al. ${ }^{14}$ and Gliem et al., ${ }^{9}$ we demonstrated that pPLR latencies were 
increased by $20 \mathrm{msec}$; these values reached significancy $(p<0.01)$ in the DNP group, i.e. $44 \%$ of all DM patients.

Anything affecting the visual pathway may result in changes in pupillary latency. In a group of DM patients reported by Lanting et al. ${ }^{4,18-20}$ the visual evoked potential latencies were in the majority of patients within a normal range. Lanting et al. concluded that in DM patients the increased pPLR latencies primarily reflect an efferent pupillary defect and prolongation of the pPLR latency would be largely caused by autonomic dysfunction.

In the normal pupil, the range of light levels that reach the retina at different frequencies reduce the phase lag and the equivalent latency of the according pupil responses. This latency is independent of target distance and pupil size. ${ }^{9,21}$ When pupillary responses are classified by the size of the response, rather than by the size of the stimulus, the latencies of the larger responses to light stimuli are shorter than those of the smaller responses (Figure 5). Exceptions occur, when initial pupil size is small, which restrict the size of the response. ${ }^{9}$ From the "pupil size effect" we know that a signal dependent on the static pupil size regulates the gains of the parallel phasic and tonic pathways, the former being responsive to transient changes of light. The presumptive locus of this mechanism or regulator function likely resides near the Westphal Edinger (WE) nucleus. ${ }^{9,11}$

The pupil gain was highly significantly diminished, mainly for lower frequencies, in the DNP patient group who showed at least two clinical signs of neuropathy, but not so in the overall patient group. This is probably a sign of the decreased dynamic flexibility of the pupil in diabetic patients with neuropathy due not only to the less flexible, diminished neural input but also due to the changes in structural anatomical muscle dynamics known to take place in diabetic patients after some time, especially when there is also a neuropathy. ${ }^{11,12,15}$

The pupil phase of the patients varied with respect to the control group. For the lower frequencies of 0.1 and $0.3 \mathrm{~Hz}$ in the DNP patients, we found a highly significant phase lead that was only a tendency within the group of all patients. For higher frequencies of 0.7 and 1.3 $\mathrm{Hz}$, however, we found a significant phase lag, especially in the DNP patients as compared to normal subjects.

The phase lead for lower frequencies could be due to long-term adaptation to the neuropathy and might be generated by cerebellar ${ }^{14}$ control of the pupil. Similarly, the phase lag for higher frequencies might be due to different cerebellar modulation of the midbrain dynamic pupil reflex that may show a different control characteristic of the midbrain centers, ncl. Westphal Edinger [WE] by the cerebellum, ${ }^{22}$ or by the olivary pretectal nucleus. ${ }^{4,23}$

Our comparison of the frequency distribu-
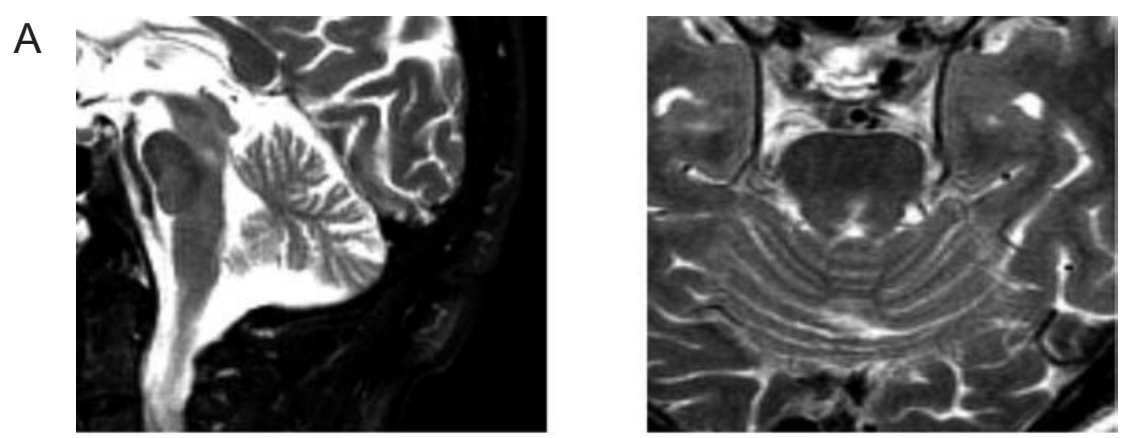

B

\begin{tabular}{lcccccc} 
Diameter & $\begin{array}{c}\text { Pupil } \\
\text { Contract }\end{array}$ & $\begin{array}{c}\text { Vmax } \\
\text { Dilation } 1\end{array}$ & $\begin{array}{c}\text { Vmax } \\
\text { Contract }\end{array}$ & Amplit. & Gain & Phase $\left({ }^{\circ}\right)$ \\
Pat. $4288-05$ & $4.25 \mathrm{~mm}$ & $3.29 \mathrm{~mm} / \mathrm{s}$ & $0.91 \mathrm{~mm} / \mathrm{s}$ & $1.17 \mathrm{~mm}$ & 0.148 low F & 84.1 \\
& & & & & 0.104 high F & 149.4 \\
\hline
\end{tabular}

Figure 4. Single case example of a 59-year old male diabetic patient with autonomous polyneuropathy, who had suffered from a left dorso-paramedian ponto-mesencephalic lacunar stroke. (A) cMRT. (B) Values of his most important pupil recording parameters.

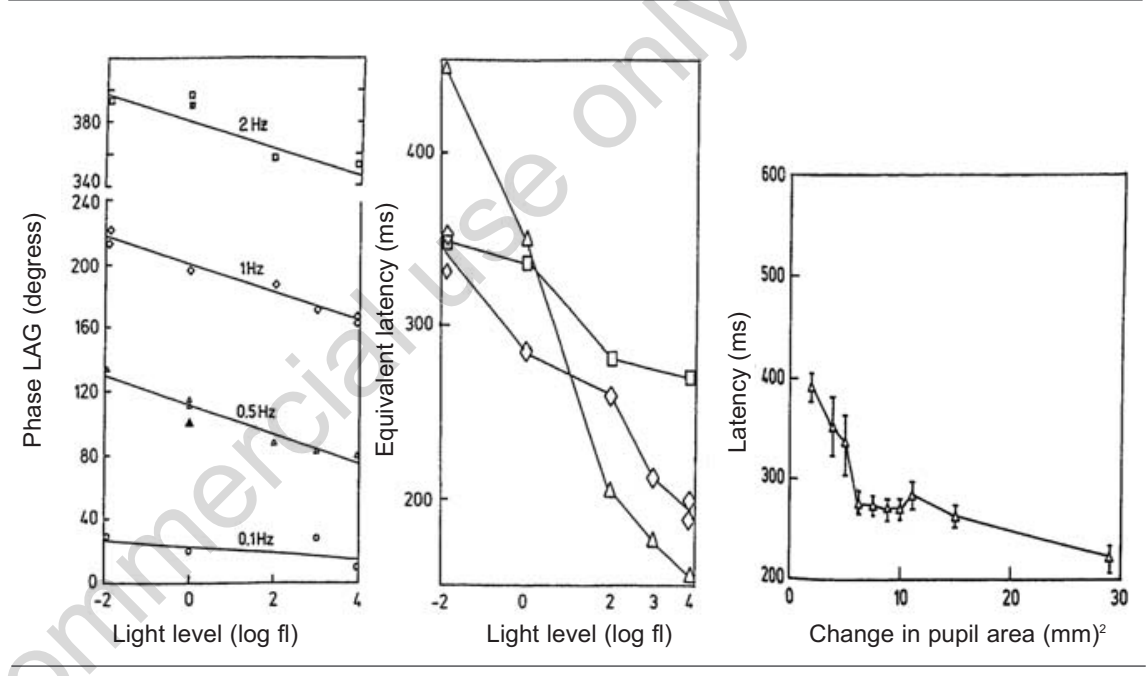

Figure 5. (Modified from Myers et al. ${ }^{21}$. Phase lag and equivalent latency of pupil responses to sinusoidal light stimuli are reduced by increased mean light level. Left: phase lag data. Middle: corresponding equivalent latencies computed from phase lag by subtracting the minimum phase component then dividing by angular frequency in $\%$ sec; subj. JCA. Right: latency of pPLR as function of response size. Note that in the light-pupil responses the response size increases with increasing stimulus size. Results are for subject GM, 20 responses per class.

tion over time using short time fourier transformation analysis (STFT) showed the abnormal decrease of low frequency variability in DM patients' phasic pupillary reflex and a significantly larger amount of high frequency content during both pPLR phases, contraction and dilation, probably due to greater stiffness of the iris muscle for both biomechanical and neural control reasons. This might be an analogy to the abnormal cardiac frequency-stability in these patients that is due to a lack of flexibility of the central autonomic innervation.

\section{Specifics of pupil innervation}

The innervation of the pupil is dominated by the parasympathetic innervation as compared to the sympathetic innervation. The relatively weak muscle which is embedded in the iris stroma represents the pupillary dilator. Therefore, the PLR is dominated by the sphincter muscle, which receives predominantly parasympathetic input. However, the central sympathetic inhibition of the neural activity at the level of the WE nucleus might also play a major role in the control of the pupil response.

The hypothesis that the sympathetic innervation may be the main factor of change in diabetic neuropathy and the change in PLR appears to be at least problematic., ${ }^{4,6,11,23}$ However, pharmacological studies have 
demonstrated that, especially in diabetes patients, there is a hypersensitivity to direct sympathicomimetics, which is probably due to earlier denervation of sympathetic fibers..$^{24,25}$ Of course, these pharmacological studies are problematic as it is very difficult to quantify the influence of different permeabilities of the corneas.

Another factor may be, as Fujii et al. ${ }^{25}$ have demonstrated, that morphological changes occur more frequently in patients with diabetes mellitus at the musculus dilatator pupillae than in the musculus sphincter pupillae. ${ }^{10}$ However, the studies by Hreidarsson et al. ${ }^{12}$ and Barron et al. ${ }^{3}$ have shown that a reduced elasticity of the pupil may not be the main factor for the above results. In general, the direct influence of the sympathetic innervation of the pupil in the diabetic autonomic neuropathy has been demonstrated very well in many studies of the cardiovascular systems. ${ }^{3,18,26-29}$ Although it is interesting to look at the two different parts of the velocities of the dilation phase of the pPLR, it has to be noted that these values show a large standard deviation both in our group of diabetic patients and in the control group. Therefore, an exact differentiation of the parasympathetic and the sympathetic deficits in cases of diabetic neuropathy using these two parameters is quite difficult and, for the above noted reasons, often questionable. ${ }^{8,11,15,23}$ The firing rate of the pupilloconstrictor neurones in the WE nucleus is high in the absence of external influence ${ }^{28}$ and pupil size may be small. A decrease in central inhibition with age is regarded to be the mechanism behind the well known age-related decrease in pupil size, especially in darkness. ${ }^{11,30}$ Here, the inhibitory inputs to the WE nucleus (e.g. from the olivary pretectal nucleus ${ }^{23}$ ) are presumed to become less effective with age. The question arises as to which factor might probably contribute most to our main finding, the decreased initial pupil size after dark adaptation in the diabetes group. Is it the peripheral change of the iris muscle plus the effect of the vegetative neuropathy? Or is it the centrally increased sympathetic inhibition of the WE nucleus innervation due to microvascular brain stem deficits in DM? This would be at least as likely as the former hypothesis since in DM patients microinfarcts which include the ponto-mesencephalic region of the 3rd nucleus and the WE nucleus ${ }^{31}$ occur relatively often, as shown in our case example (Figure 4). In conclusion, our study has demonstrated that a significantly decreased pupil diameter is an early sign of autonomic neuropathy. Additional deficits of the PLR are often to be found in cases where other symptoms of autonomic neuropathies occur. As far as these changes reflect changes in pupil dynamics they are related to the initial pupillary diameter. From our results, we conclude that in those
DM patients a vegetative neuropathy is present where the pPLR shows deficits of maximum contraction and dilation 1 velocity, where the change of the course of frequency content in the FFT and where cPLR gain and phase changes are present. However, it has to be accompanied by other signs of autonomic neuropathy in order to evaluate the final diagnosis and describe the course of autonomic neuropathy. Only together with continuously modulated light stimulation of the pupil as shown by Hultborn et al..$^{22}$ and Myers et al. ${ }^{21}$ can the early diagnosis of autonomic neuropathy be confirmed. Probably, both the sympathetic and the parasympathetic nervous system are affected by diabetic autonomic neuropathy, which likely also includes central, ponto-mesencephalic changes, although the pPLR differentiates in general between DM patients and healthy subjects; only the recording of cPLR, together with the STFT of the pPLR, allows significant deficits of pupillary control to be identified more clearly in single cases. The fact that the combination of pPLR and cPLR measurements which reflect afferent and efferent deficits that may occur in DM patients so efficiently differentiates between pathological and healthy cases appears to be an advantage for the differentiation of single cases of diabetes mellitus.

\section{References}

1. Alexandridis E, Hain G. Frequenz der flimmerlichtabhängigen Pupillen-oszillationen bei Diabetikern. Fortschr Ophthalmol 1985;82:187-8.

2. Alio J, Hernandez I, Millan A, Sanchez J. Pupil responsiviness in diabetes mellitus. Ann Ophthalmol 1989;21:132-7.

3. Barron SA, Rogovski Z, Kanter Y, Hemli Y. Parasympathetic autonomic neuropathy in diabetes mellitus: the heart is denervated more often than the pupil. Electromyogr Clin Neurophysiol 1994;34:467-9.

4. Sun F, Tauchi P, Stark L. Dynamic pupillary response controlled by the pupil size effect. Exp Neurol 1983;82:313-24.

5. Fulk GW, Bower A, McBride K, Boatright R. Sympathetic denervation of the iris dilator in noninsulin-dependent diabetes. Optom Vis Sci 1991;68:954-6.

6. Gliem H. Pupillomotorische Veränderungen bei Diabetikern. Klin Monatsbl Augenheilkd 1972;160: 293-5.

7. Heller PH, Perry F, Jewett DL, Levine JD. Autonomic components of the human pupillary light reflex. Invest Ophthalmol Vis Sci 1990;31:156-62

8. Karavanaki K, Davies AG, Hunt LP et al. Pupil size in diabetes. Arch Dis Childhood 1994;71:511-5.

9. Wilhelm BJ, Wilhelm H, Moro S, Barbur L.
Pupil response components: studies in patients with parinaud's syndrome. Brain 2002;125:2296-307.

10. Winn B, Whitaker D, Elliot DB, Phillips NJ. Factors affecting light adapted pupil size in normal human subjects. Invest Ophthalmol Vis Sci 1994;35:1132-7.

11. Pfeiffer MA, Cook D, Brodsky J et al. Quantitative evaluation of sympathetic and parasympathic control of iris function. Diabetes Care 1982;518-28.

12. Hreidarsson AB. Pupil Motility in longterm diabetes. Diabetologica 1979;17:14550.

13. Hreidarsson AB. Pupil size in insulindependent diabetes. Relationship to duration, metabolic control, and long-term manifestations. Diabetes 1982;31: 442-8.

14. Hreidarsson AB, Gunderson HJG. The pupillary response to light in type 1 (insulin-dependent) diabetes. Diabetologica 1985;28:815-21.

15. Keane JR, Ahmadi J. Most diabetic third nerve palsies are peripheral. Neurology 1998;51:1510.

16. Straub RH, Jeron A, Kerp L. The pupillary light reflex. 2. Prevalence of pupillary autonomic neuropathy in diabetics using agedependent and age-independent pupillary parameters. Ophthalmologica 1992;204: 143-8.

17. Niakan E, Harati Y, Comstock JP. Diabetic autonomic neuropathy. Metabolism 1986;35:224-34.

18. Lanting P, Bos JE, Aartsen J, Schuman L, et al. Assessment of pupillary light reflex latency and darkness adapted pupil size in control subjects and diabetic patients with and without cardiovascular autonomic neuropathy. J Neurology Neurosurgery and Psych 1990;53:912-4.

19. Lanting P, Strijers RLM, Bos JE, et al. The cause of increased pupillary light reflex latencies in diabetic patients: the relationship between pupillary light reflex and visual evoked potential latencies. Electroencephalogr Clin Neurophysiol 1991;78: 111-5.

20. Murakami M, Kitano I, Hitoshi Y, Ushio Y. Isolated oculomotor nerve palsy following midbrain infarction. Clin Neurol Neurosurgery 1994;96:188-90.

21. Myers GA, Gannon JA, Stark LW. Level dependent signal flow in the light pupil reflex. II. Phase velocity of responses to sinusoidal light stimuli. Biol Cybern 1993;68: 235-40

22. Hultborn H, Mori K, Tsukahara N. Cerebellar influence on parasympathetic neurones innervating intraoccular muscles. Brain Res 1978;159:269-78.

23. Breen LA, Hopf HC, Farris BK, Gutmann L. Pupil-sparing oculomotor nerve palsy due to midbrain infarction. Arch Neurol 1991; 
48:105-6.

24. Clarke RJ, Ikeda H. Properties of neurones in the nucleus of the optic tract of the pretectum in the cat [proceedings]. J Physiol $1978 ; 275: 46-7$

25. Fujii T, Ishikawa S, Uga S. Ultrastructure of iris muscles in Diabetes mellitus. Ophthalmologica Basel 1977;174:228-39

26. Zinn K. Anatomy of the Iris. In: Zinn K. The Pupil. Springfield. Illinois: Charles C
Thomas 1972:3-33.

27. McDougall AJ, McLeod JG. Autonomic neuropathy. I. Clinical features, investigation, pathophysiology, and treatment. J Neurolog Sci 1996;137:79-88.

28. Saeki N, Murai H, Mine S, Yamaura A. Fascicular arrangement within the oculomotor nerve MRI analysis of a midbrain infarct. J Clin Neurosci 2000;7:268-70.

29. Sillito R, Zbrozyna AW. The activity charac- teristics of the preganglionic pupilloconstrictor neurones. J Physiol 1970;211:769-79.

30. Loewenfeld IE. Pupillary changes related to age. In: Thompson HS, Daroff R, Frisen L, Glaser JS, Sanders MD editors. Topics in Neuro-ophthalmology. Baltimore: Williams \& Wilkins 1972 p.124-50. 31.Hopf HC, Gutmann L. Diabetic 3rd nerve palsy: Evidence for a mesencephalic lesion. Neurology 1990;40:1041-5. 